\section{PO-0778 WITHDRAWN}

\section{PO-0779 WHICH PARAMETERS ARE MANDATORY IN URINARY TRACT INFECTION IN CHILDREN?}

M Neamtu, L Dobrota. Pediatric Clinic, Pediatric Clinic Hospital "Lucian Blaga" University of Sibiu, Sibiu, Romania

\subsection{6/archdischild-2014-307384.1416}

Background The protocol of investigations in urinary tract infection (UTI) in children mandatory includes urianalysis, uroculture, reactive $\mathrm{C}$ protein (RCP), blood urea (BUN), renal ultrasonography and scintigraphy. Each one of these has limits to perform in children.

Aims To establish the correlations between UTI investigations for argumentation the therapeutic decision.

Methods Retrospective study of hospitalised paediatric patients for UTI. The study parameters were: age, symptoms, RCP, BUN, uroculture, renal ultrasonography, urinanalysis (leukocytes, nitrites, $\mathrm{pH}$, density, proteins, erythrocytes, ascorbic acid-AA).

Results Were studied 243 hospitalised and treated with antibiotics UTI. Uroculture was positive in 178 patients (143 E.coli, 16 Enterococcus, 17 Proteus, 2 Staphylococcus). Negative uroculture was considered as decapitated UTI by antibiotics initiated before admission. 52 patients were $<1$ year old. Fever has been presented in 204 patients. 96 (56,1\% from patients $>1$ year old) presented renal specific symptoms. 22 (42,3\% from patients $<1$ year old) presented unspecific symptoms (vomiting, diarrhoea). 84 presented high values of RCP and 25 of BUN, 104 significant leukocyturia, 12 nitrites, 211 AA, 24 abnormal ultrasonography. Fever was the most important clinical parameter in younger patients (p 0,03), while unspecific symptoms were not significantly more frequent $(\mathrm{p} 0,25)$; the majority of patients with high

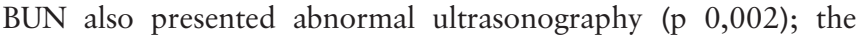
reduced frequency of nitrites was due to AA (p 0,0001). Negative uroculture has no diagnostic significance ( $\mathrm{p} 0,78)$, only in presence of significant leukocyturia and high RCP.

Conclusions In guideline of UTI diagnosis with negative uroculture, fever, RCP, BUN, leukocyturia, ultrasonography become mandatory.

\section{P0-0780 POSTNATAL FOLLOW-UP OF NEWBORNS WITH PRENATAL DIAGNOSIS OF HYDRONEPHROSIS}

${ }^{1} S$ Foldi, ${ }^{1} M$ Feldman, ${ }^{1} E$ Nadir, ${ }^{2} R$ Krawitsky, ${ }^{3} R$ Yakubowich. ${ }^{1}$ Department of Neonatology, Hillel Yaffe Medical Center, Hadera, Israel; ${ }^{2}$ Department of Radiology, Hillel Yaffe Medical Center, Hadera, Israel; ${ }^{3}$ Department of Pediatrics, Hillel Yaffe Medical Center, Hadera, Israel

\subsection{6/archdischild-2014-307384.1417}

Background Prenatal Hydronephrosis is diagnosed in 1-5\% of pregnancies worldwide. The diagnosis of antenatal hydronephrosis $(\mathrm{ANH})$ causes stress to the parents and dilemmas to the paediatrician. Objectives To examine the correlation between the degree of the renal pelvic dilatation (RPD) detected by the first $2-5$ days of life and the postnatal outcome. To investigate the correlation between bilateral hydronephrosis and the nephrologic outcome. To discuss the possibility of decreasing the postnatal examinations of these healthy babies.

Methods During a period of two years, we enrolled 143 term newborns with ANH. These babies had an ultrasound at the age of 2-5 days and a second ultrasound at the age of 4-6 weeks. After the examinations they were referred to our nephrologist.
Results Out of 8370 live-births, 143 infants had ANH. Six babies never completed the exam. At the first exam 69 babies were normal, 62 babies had mild, 3 babies had moderate and 3 babies had severe RPD. On their second ultrasound 76 had normal findings, 36 had mild, 8 had moderate and 12 had severe RPD. 132 babies completed both of the examinations. Bilateral hydronephrosis was detected in 33 cases during their first ultrasound while on the second only 27 . There were 11 infants with UPJ obstruction 9 of them with severe RPD. We found 10 babies with VUR. Six babies needed surgical intervention.

Conclusions There seems to be a correlation between the degree of RPD and the presence of postnatal pathology. Bilateral hydronephrosis probably carries increased risk for postnatal pathology.

\section{PO-0781 HIVAN IN A YOUNG CAUCASIAN FEMALE}

${ }^{1} \mathrm{M}$ Gafencu, ${ }^{1} \mathrm{G}$ Doros, ${ }^{2} \mathrm{R}$ Costa. ${ }^{1}$ Pediatrics, "Victor Babes" University of Medicine and Pharmacy, Timisoara, Romania; ${ }^{2}$ Pediatrics, Children Hospital Timisoara, Timisoara, Romania

\subsection{6/archdischild-2014-307384.1418}

Background Renal impairment in patients with HIV/AIDS is described since the beginning of pandemia. The incidence is increasing in the last 10 years by associated comorbidities: hypertension, diabetes, lipids disorders. In Romania there is a cohort (those born 1988-1990) around 9000 HIV -infected patients over 20 years old, which started antiretroviral therapy (ART) since 1996/1997.

Methods We studied an HIV case - white female, 22 years, from the nephrological perspective, with all complex comorbidities.

Results Our patient was diagnosed with AIDS at 11 years when she was admitted with toxoplasmic encephalitis, comatose. Since 2000 with ART. After that started ART side effects: hypercholesterolemia, hypertension, cardiomyopathy and an early renal failure. Renal impairment was detected at the age of 15 year with the GFR estimation (MDRD)-61 $\mathrm{ml} / \mathrm{min}$, with elevated blood pressure values. Treatment of complications was correction of dyslipidemia (Pravastatin + Ezetrol), intermittently diuretics, and from 2009 - Captopril. From 2012 she is in the C2 stage of HIV infection, RNA undetectable, CKD stage III (GFR-MDRD $36 \mathrm{ml} / \mathrm{min}$ ). The renal biopsy performed revealed chronic glomerulopathy and diffuse global glomerulosclerosis. She present now osteopenia and a neurologic sequel (hemiparesis).

Conclusions Renal pathology is found in all stages of HIV infection. Biopsy is one that correctly diagnose the case even if the lesions are not patognomonic for HIVAN. The case is with a complex pathology related with the HIV infection. Renal impairment, a fact in these patients, often need renal replacement therapy (dialysis or renal transplantation), less than in US population confronted with a higher prevalence of HIVAN.

\section{PO-0782 WITHDRAWN}

\section{PO-0783 CONTRIBUTION OF CYSTOGRAPHY IN INFANTS WITH FIRST URINARY TRACT INFECTION}

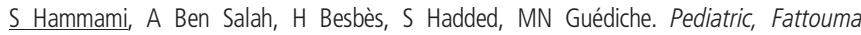
Bourguiba Hospital, Monastir, Tunisia

\subsection{6/archdischild-2014-307384.1419}

Objectives To assess the yield of radiological retrograde uretrocystography (UCR) done systematically after a first episode of urinary tract infection (UTI) in infant less than 1 year. 